\title{
A General Sparse Image Prior Combination in Super-Resolution
}

\author{
Salvador Villena*, Miguel Vega*, Rafael Molina ${ }^{\dagger}$ and Aggelos K. Katsaggelos ${ }^{\ddagger}$ \\ *Dpto. de Lenguajes y Sistemas Informáticos \\ Universidad de Granada, Granada 18071, SPAIN \\ Emails: \{svillena,mvega\}@ugr.es \\ ${ }^{\dagger}$ Dpto. de Ciencias de la Computación e Inteligencia Artificial \\ Universidad de Granada, Granada 18071, SPAIN \\ Email:rms@decsai.ugr.es \\ $\ddagger$ Department of Electrical and Computer Engineering \\ Northwestern University, Evanston, IL 60208, USA \\ Email: aggk@eecs.northwestern.edu
}

\begin{abstract}
In this paper the Super-Resolution (SR) image registration and reconstruction problem is studied within the Bayesian framework using a general sparse image prior combination. The representation of the proposed priors as Scale Mixtures of Gaussians (SMG), leads to the introduction of variational parameters, for which degenerate distributions are assumed. In the proposed method all the problem unknowns are automatically estimated using variational techniques. An experimental comparison between the proposed and state of the art methods has been performed, on both synthetic and real images.

Index Terms-image processing; superresolution;
\end{abstract}

\section{INTRODUCTION}

Image SR is the process of obtaining a High Resolution (HR) image from a set of degraded Low Resolution (LR) images (see [1], [2] for a review). The basic principle in $\mathrm{SR}$ is that changes in LR images caused by the blur and the camera (and/or scene) motion provide additional information that can be utilized to reconstruct the HR image. Usually SR methods include two parts: registration, where the motion between LR images, or updated versions of them, is estimated, and image reconstruction, where the HR image is recovered from the LR images. In this paper, both registration and reconstruction are studied within the Bayesian framework. In the Bayesian framework a prior model on the HR image to be reconstructed is introduced. Its aim is to encapsulate our prior image knowledge and consequently to avoid the ill-posedness of the image reconstruction problem.

Sparse image priors are known to produce good results in Bayesian image restoration, in general, and in SR, in particular (see [3], [4]). An image prior is considered as sparse when it is Super-Gaussian (SG) [5], i.e., compared to the Gaussian distribution, it has heavier tails and it is more peaked. These distributions are referred to as sparse since most of the distribution mass is located around zero (hence strongly favoring zero values), but the probability of

This work was supported in part by the Comisión Nacional de Ciencia y Tecnología under contract TIN2010-15137 and microproyect cod.21 CeiBiotic of University of Granada. occurrence of large signal values is higher compared to the Gaussian distribution. Recently, a new general formalism for SG image priors has been proposed and successfully applied to the blind deconvolution problem [6]. In this paper we explore the application of the general formalism proposed in [6] to the SR problem and propose the use of a product of independent Gaussian distributions as the image prior. This representation will allow us to estimate all the unknowns of the SR problem.

The rest of this paper is organized as follows. Section II provides the mathematical model for the LR image acquisition process. We provide the description of the hierarchical Bayesian framework modeling the unknowns in Section III. The inference procedure to develop the proposed methods is presented in Section IV. We demonstrate the effectiveness of the proposed methods with experimental results in Section $\mathrm{V}$ and conclusions are drawn in Section VI.

\section{Problem Formulation}

Let us consider an imaging process that generates an LR image set $\left\{\mathbf{y}_{k}\right\}=\mathbf{y}_{1}, \ldots, \mathbf{y}_{L}$ from the HR image $\mathbf{x}$. The LR images $\mathbf{y}_{k}$ and the HR image $\mathbf{x}$ consist of $N$ and $P N$ pixels, respectively, where the integer $P>1$ is the increasing resolution factor. In this paper we adopt a matrix-vector notation, that is, images $\mathbf{y}_{k}$ and $\mathbf{x}$ are arranged as $N \times 1$ and $P N \times 1$ vectors, respectively. The imaging process introduces warping, blurring and downsampling, which is modeled as

$$
\mathbf{y}_{k}=\mathbf{A} \mathbf{H}_{k} \mathbf{C}\left(\mathbf{s}_{k}\right) \mathbf{x}+\mathbf{n}_{k}=\mathbf{B}\left(\mathbf{s}_{k}\right) \mathbf{x}+\mathbf{n}_{k},
$$

where $\mathbf{A}$ is the $N \times P N$ downsampling matrix, $\mathbf{H}_{k}$ the $P N \times P N$ blurring matrix, $\mathbf{C}\left(\mathbf{s}_{k}\right)$ the $P N \times P N$ warping matrix generated by the motion vector $\mathbf{s}_{k}$, and $\mathbf{n}_{k}$ is the $N \times 1$ acquisition noise. We assume that the blurring $\mathbf{H}_{k}$ matrices are known. The effects of downsampling, blurring, and warping can be combined into a single $N \times P N$ system matrix $\mathbf{B}\left(\mathbf{s}_{k}\right)$. Given Eq.(1), the super resolution problem is to find an estimate of the HR image $\mathbf{x}$ from the set of LR images $\left\{\mathbf{y}_{k}\right\}$ using prior knowledge about $\left\{\mathbf{n}_{k}\right\}$ and $\mathbf{x}$. 
Usually the motion vector set $\left\{\mathbf{s}_{k}\right\}$ is not known, so it has to be estimated along with the HR image $\mathbf{x}$. We consider a motion model consisting of translational and rotational motion, that is, $\mathbf{s}_{k}=\left(\theta_{k}, h_{k}, v_{k}\right)^{T}$, where $\theta_{k}$ is the rotation angle, and $h_{k}$ and $v_{k}$ are respectively the horizontal and vertical translations of the $k^{t h} \mathrm{HR}$ image with respect to the reference frame $\mathbf{x}$. The detailed description of the explicit form of the $\mathbf{C}\left(\mathbf{s}_{k}\right)$ matrices can be found in [4].

\section{Hierarchical Bayesian Models}

In the following subsections we provide the description of individual distributions used to model the unknowns.

\section{A. Observation Model}

Using the model in Eq.(1), assuming zero-mean white Gaussian noise $\mathbf{n}_{k}$ with inverse variance (precision) $\beta_{k}$, and statistical independence of the noise among the LR image acquisitions, the conditional probability of the set of LR images $\left\{\mathbf{y}_{k}\right\}$, given $\mathbf{x}$, the motion vectors $\left\{\mathbf{s}_{k}\right\}=\left\{\mathbf{s}_{1}, \ldots, \mathbf{s}_{L}\right\}$, and $\left\{\beta_{k}\right\}=\left\{\beta_{1}, \ldots, \beta_{L}\right\}$ can be expressed as

$$
\mathrm{p}\left(\left\{\mathbf{y}_{k}\right\} \mid \mathbf{x},\left\{\mathbf{s}_{k}\right\},\left\{\beta_{k}\right\}\right)=\prod_{k=1}^{L} \mathcal{N}\left(\mathbf{y}_{k} \mid \mathbf{B}\left(\mathbf{s}_{k}\right) \mathbf{x}, 1 / \beta_{k}\right) .
$$

\section{B. A General Sparse Image Prior Combination}

In this paper we utilize the following combination of general sparse priors

$$
\mathrm{p}(\mathbf{x})=\prod_{j=1}^{d} \prod_{i=1}^{P N} \mathrm{p}\left(z_{j}(i)\right),
$$

on the unknown filtered images set $\{\mathbf{z}\}=\left\{\mathbf{z}_{1}, . ., \mathbf{z}_{d}\right\}$, where $\mathbf{z}_{j}=\mathbf{F}_{j} \mathbf{x}$, and $\mathbf{F}_{j}$ are convolution operators. In Eq. (3) $\left.z_{j}(i)\right)$ denotes the $i$ component of the $\mathbf{z}_{j}$ vector. Notice that in Eq. (3) we are approximating the partition function as an independent product of partition functions.

The general sparse priors $\mathrm{p}\left(z_{j}(i)\right)$ of Eq.(3) are defined as

$$
\mathrm{p}\left(z_{j}(i)\right)=\gamma \exp \left(-\rho\left(z_{j}(i)\right)\right),
$$

where $\gamma$ is a normalization constant, that is $\gamma^{-1}=$ $\int \exp [-\rho(u)] \mathrm{d} u$, and $\rho($.$) is a penalty function symmetric$ around 0 (see [6]). Sparsity is achieved when the function $\rho$ leads to the suppression of most coefficients $z_{j}(i)$ while preserving a small number of important features.

Formally, for $\mathrm{p}(u)=\gamma \exp [-\rho(u)]$ to be $\mathrm{SG}$, the function $\rho(\sqrt{s})$ has to be increasing and concave for $s \in(0, \infty)$ [5]. This condition is equivalent to $\rho^{\prime}(s) / s$ being decreasing on $(0, \infty)$. This allows us to express $\mathrm{p}\left(z_{j}(i)\right)$ of Ec. (4) as an SMG, i.e.,

$$
\mathrm{p}\left(z_{j}(i)\right)=\int \mathcal{N}\left(z_{j}(i) \mid 0,1 / \xi\right) \mathrm{p}(\xi) \mathrm{d} \xi .
$$

Using the SMG representation, and introducing the new variables $\left\{\boldsymbol{\eta}_{d}\right\}=\left\{\boldsymbol{\eta}_{1}, . ., \boldsymbol{\eta}_{d}\right\}$, with $\boldsymbol{\eta}_{j} \in R^{+P N}$, it is possible to transform the general sparse prior $\mathrm{p}(\mathrm{x})$ of $\mathrm{Eq}$.(3) to the Gaussian form

$$
\mathrm{p}\left(\left\{\boldsymbol{\eta}_{d}\right\}, \mathbf{x}\right)=\prod_{j=1}^{d} \prod_{i=1}^{P N} \mathcal{N}\left(z_{j}(i) \mid 0,1 / \eta_{j}(i)\right) \mathrm{p}\left(\eta_{j}(i)\right) .
$$

In this paper we use the following image prior

$$
\mathrm{p}\left(\left\{\boldsymbol{\eta}_{d}\right\}, \mathbf{x}\right)=\prod_{j=1}^{d} \prod_{i=1}^{P N} \mathcal{N}\left(z_{j}(i) \mid 0,1 / \eta_{j}(i)\right),
$$

which can be written as

$$
\mathrm{p}\left(\left\{\boldsymbol{\eta}_{d}\right\}, \mathbf{x}\right)=\prod_{j=1}^{d} \prod_{i=1}^{P N} \mathcal{N}\left(z_{j}(i) \mid 0,1 / \eta_{j}(i)\right) \mathrm{p}\left(\eta_{j}(i)\right),
$$

where $\mathrm{p}\left(\eta_{j}(i)\right)$ is for all $i$ and $j$ a degenerate distribution.

This SMG representation allows us to easily perform inference using a variational procedure, (see [6], for details).

\section{Hyperpriors on the Hyperparameter}

The hyperparameters $\left\{\beta_{k}\right\}$ are crucial for the performance of the SR algorithm. For their modeling, we employ Gamma distributions

$$
\mathrm{p}\left(\left\{\beta_{k}\right\}\right)=\prod_{k=1}^{L} \Gamma\left(\beta_{k} \mid a_{\beta_{k}}^{o}, b_{\beta_{k}}^{o}\right),
$$

where $a_{\beta_{k}}^{o}>0$ and $b_{\beta_{k}}^{o}>0$ are the shape and scale parameters, respectively. The hyperpriors are chosen as Gamma distributions since they are conjugate priors for the Gaussian distribution.

\section{Modeling the uncertainties in the registration parameters}

Let us denote by $\left\{\overline{\mathbf{s}}^{p}\right\}$ the estimate of $\left\{\mathbf{s}_{k}\right\}=\left\{\mathbf{s}_{1}, \ldots, \mathbf{s}_{L}\right\}$ obtained from LR observations in a preprocessing step, using registration algorithms, such as the ones reported in [7]. As these estimates are in general inaccurate, we model the motion parameters, as stochastic variables following Gaussian distributions with a priori means set equal to the preliminary motion parameters $\overline{\mathbf{s}}_{k}^{p}$, that is,

$$
\mathrm{p}\left(\left\{\mathbf{s}_{k}\right\}\right)=\prod_{k=1}^{L} \mathcal{N}\left(\mathbf{s}_{k} \mid \overline{\mathbf{s}}_{k}^{p}, \mathbf{\Xi}_{k}^{p}\right),
$$

with $\boldsymbol{\Xi}_{k}^{p}$ the a priori covariance matrix. The parameters $\overline{\mathbf{s}}_{k}^{p}$ and $\boldsymbol{\Xi}_{k}^{p}$ incorporate prior knowledge about the motion parameters into the estimation procedure. If such knowledge is not available, $\overline{\mathbf{s}}_{k}^{p}$ and $\left(\boldsymbol{\Xi}_{k}^{p}\right)^{-1}$ can be set equal to zero, which makes the observations solely responsible for the estimation process.

\section{E. Joint Model}

Combining Eqs. (2),(8),(9)\&(10) we obtain the following joint probability distribution

$$
\begin{aligned}
\mathrm{p}\left(\Theta,\left\{\mathbf{y}_{k}\right\}\right)= & \mathrm{p}\left(\left\{\mathbf{y}_{k}\right\} \mid \mathbf{x},\left\{\mathbf{s}_{k}\right\},\left\{\beta_{k}\right\}\right) \times \\
& \mathrm{p}\left(\left\{\boldsymbol{\eta}_{d}\right\}, \mathbf{x}\right) \mathrm{p}\left(\left\{\mathbf{s}_{k}\right\}\right) \mathrm{p}\left(\left\{\boldsymbol{\eta}_{d}\right\}\right) \mathrm{p}\left(\left\{\beta_{k}\right\}\right),
\end{aligned}
$$

where $\Theta=\left\{\left\{\boldsymbol{\eta}_{d}\right\}, \mathbf{x},\left\{\mathbf{s}_{k}\right\},\left\{\beta_{k}\right\}\right\}$ denotes the set of all unknowns. 


\section{VARIATIONAL BAYESIAN INFERENCE}

The Bayesian inference is based on the posterior distribution $p(\Theta \mid \mathbf{y})$ which can be variationally approximated by the $q(\Theta)=\prod_{\zeta \in \Theta} q(\zeta)$ distribution, where the unknown distributions $\mathrm{q}(\zeta), \zeta \in \Theta$ are obtained from

$$
\mathrm{q}(\zeta) \propto \exp \left(\langle\log [\mathrm{p}(\Theta, \mathbf{y})]\rangle_{\Theta_{\zeta}}\right),
$$

where $\Theta_{\zeta}$ denotes the set $\Theta$ with $\zeta$ removed, and $\mathrm{E}_{\mathrm{q}\left(\Theta_{\zeta}\right)}[\cdot]=<\cdot>_{\Theta_{\zeta}}$. In the following, the subscript of the expected value will be removed when it is clear from the context.

From Eq.(12), we obtain for $q(\mathbf{x})$

$$
\begin{array}{r}
\mathrm{q}(\mathbf{x}) \propto \exp \left\{\mathrm { E } \left[\log \left(\mathrm{p}\left(\left\{\mathbf{y}_{k}\right\} \mid \mathbf{x},\left\{\mathbf{s}_{k}\right\},\left\{\beta_{k}\right\}\right)\right]_{\left\{\mathbf{s}_{k}\right\}}\right.\right. \\
+\mathrm{E}\left[\log \left(\mathrm{p}\left(\left\{\boldsymbol{\eta}_{d}\right\}, \mathbf{x}\right)\right]_{\left\{\boldsymbol{\eta}_{d}\right\}}\right\},
\end{array}
$$

which is the multivariate Gaussian $\mathrm{q}(\mathbf{x})=\mathcal{N}\left(\mathbf{x} \mid \hat{\mathbf{x}}, \operatorname{cov}_{\mathrm{q}(\mathbf{x})}\right)$ with

$$
\operatorname{cov}_{\mathrm{q}(\mathbf{x})}^{-1}=\sum_{j=1}^{d} \mathbf{F}_{j}^{t} \operatorname{diag}\left(\mathbf{w}_{j}\right) \mathbf{F}_{j}+\sum_{k=1}^{L} \beta_{k} \mathrm{E}\left[\mathbf{B}\left(\mathbf{s}_{k}\right)^{t} \mathbf{B}\left(\mathbf{s}_{k}\right)\right]_{\mathbf{s}_{k}},
$$

and

$$
\hat{\mathbf{x}}=\operatorname{cov}_{\mathbf{q}(\mathbf{x})} \sum_{k=1}^{L} \beta_{k} \mathrm{E}\left[\mathbf{B}\left(\mathbf{s}_{k}\right)\right]_{\mathbf{s}_{k}}^{t} \mathbf{y}_{k} .
$$

In Eq.(14), the $\mathbf{w}_{j}$ for $j=1, \ldots, d$, are $P N$ vectors with components

$$
w_{j}(i)=\mathrm{E}\left[\eta_{j}(i)\right]_{\eta_{j}(i)}, \quad \text { for } \quad i=1, \ldots, P N,
$$

which for our degenerate $\mathrm{p}\left(\eta_{j}(i)\right)$ distributions, take the value

$$
\mathrm{E}\left[\eta_{j}(i)\right]_{\eta_{j}(i)}=1 / \mathrm{E}\left[z_{j}^{2}(i)\right]_{\mathbf{x}},
$$

with

$\mathrm{E}\left[z_{j}^{2}(i)\right]_{\mathbf{x}}=\mathrm{E}_{\mathbf{q}(\mathbf{x})}[\mathbf{x}]^{t} \mathbf{F}_{j}^{t} \mathbf{J}^{i i} \mathbf{F}_{j} \mathrm{E}_{\mathrm{q}(\mathbf{x})}[\mathbf{x}]+\operatorname{tr}\left(\operatorname{cov}_{\mathbf{q}(\mathbf{x})} \mathbf{F}_{j}^{t} \mathbf{J}^{i i} \mathbf{F}_{j}\right)$,

where $\mathbf{J}^{i i}$ is the single-entry matrix with zero everywhere except at the entry $(i, i)$, which is equal to one. The estimation of $\mathrm{E}\left[z_{j}^{2}(i)\right]_{\mathbf{x}}$, using Eq. (18), requires the evaluation of the trace of a matrix product involving the covariance matrix $\operatorname{cov}_{\mathrm{q}(\mathbf{x})}$. As this covariance matrix cannot be obtained in exact form, the Jacobi approximation has been applied in this paper.

The posterior distribution approximations $\mathrm{q}\left(\beta_{k}\right)$ are obtained from Eq. (12) as

$$
\begin{aligned}
\mathrm{q}\left(\beta_{k}\right)= & \beta_{k}^{\frac{N}{2}-1+a_{\beta_{k}}^{0} \times} \\
& \exp \left[-\beta_{k}\left(b_{\beta_{k}}^{0}+\frac{1}{2} \mathrm{E}\left[\left\|y_{k}-\mathrm{E}\left[\mathbf{B}\left(\mathbf{s}_{k}\right)\right]_{\mathbf{s}_{k}} \mathbf{x}\right\|^{2}\right]_{\mathbf{x}}\right)\right] .
\end{aligned}
$$

Also from Eq.(12) the posterior distribution approximation for $\mathrm{q}\left(\mathbf{s}_{k}\right)$ is found as

$$
\begin{aligned}
& \mathrm{q}\left(\mathbf{s}_{k}\right) \propto \exp \left(-\frac{1}{2}\left(\left\langle\beta_{k}\right\rangle \mathrm{E}\left[\left\|\mathbf{y}_{k}-\mathbf{B}\left(\mathbf{s}_{k}\right) \mathbf{x}\right\|^{2}\right]_{\mathbf{x}}\right.\right. \\
&\left.\left.+\left(\mathbf{s}_{k}-\overline{\mathbf{s}}_{k}^{p}\right)^{T}\left(\boldsymbol{\Xi}_{k}^{p}\right)^{-1}\left(\mathbf{s}_{k}-\overline{\mathbf{s}}_{k}^{p}\right)\right)\right) .
\end{aligned}
$$

The computation of $\mathrm{E}\left[\mathbf{B}\left(\mathbf{s}_{k}\right)^{t}\right]_{\mathbf{s}_{k}}$ in Eq.(15), $\mathrm{E}\left[\mathbf{B}\left(\mathbf{s}_{k}\right)^{t} \mathbf{B}\left(\mathbf{s}_{k}\right)\right]_{\mathbf{s}_{k}}$ in Eq.(14), and $\mathrm{E}\left[\left\|\mathbf{y}_{k}-\mathbf{B}\left(\mathbf{s}_{k}\right) \mathbf{x}\right\|^{2}\right]_{\mathbf{x}}$ in Eq.(20), are not straightforward since $\mathbf{B}\left(\mathbf{s}_{k}\right)$ is nonlinear with respect to $\mathbf{s}_{k}$. In [4] these estimations were performed by expanding $\mathbf{B}\left(\mathbf{s}_{k}\right)$, using its first-order Taylor series, around the mean value $\left\langle\mathbf{s}_{k}>=\overline{\mathbf{s}}_{k}=\left(\bar{\theta}_{k}, \bar{h}_{k}, \bar{v}_{k}\right)^{T}\right.$ of the distribution $\mathrm{q}\left(\mathbf{s}_{k}\right)$. We follow here the same approach, and refer to [4], where the detailed derivation and the resulting expressions for these estimated values may be found.

The proposed algorithm is summarized below in Algorithm 1.

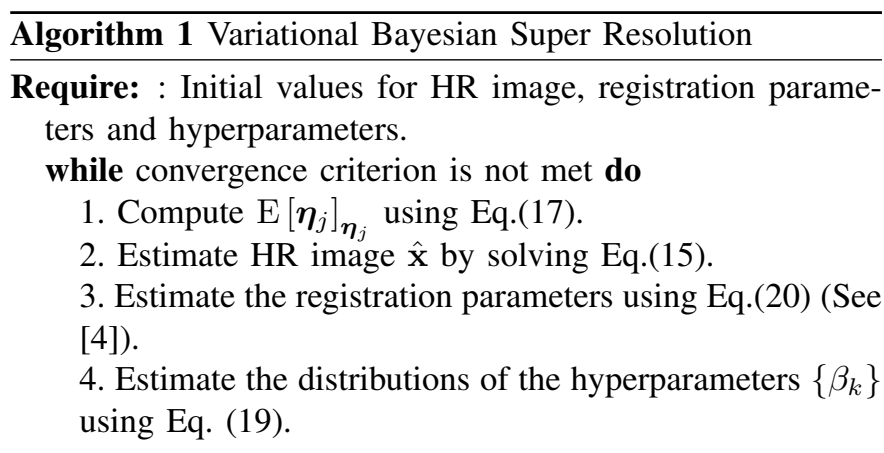

\section{EXPERIMENTAL RESULTS}

The proposed prior model of Eq. (3), allows for the combination of several filtered images $\mathbf{z}_{j}$, and in this section the following combinations have been considered: 1) NF2 combines horizontal and vertical first order differences (f.o.d.), 2) NF3 horizontal and vertical f.o.d. with the Laplacian filter, 3) NF4 horizontal, vertical and diagonal f.o.d., and 4) NF5 combines NF4 with the Laplacian filter.

In all experiments reported below, the initial values of Algorithm 1 are chosen as follows: The HR image estimate is initialized using the bicubic interpolation of observation $\mathbf{y}_{1}$. The inverse covariance matrices $\left(\boldsymbol{\Xi}_{k}^{p}\right)^{-1}$ are set equal to zero matrices, that is, no prior information is utilized about the uncertainty of motion vectors. The covariance matrices in Algorithm 1 are initially set equal to zero. The rest of the algorithm parameters are automatically calculated from the initial HR image estimate using the algorithmic steps provided in Algorithm 1. As convergence criterion we used $\left\|\mathrm{x}^{n}-\mathrm{x}^{n-1}\right\|^{2} /\left\|\mathrm{x}^{n-1}\right\|^{2}<10^{-5}$, where $\mathrm{x}^{n}$ and $\mathrm{x}^{n-1}$ are the image estimates at the $n$-th and $(n-1)$-st iterations, respectively.

Let us first perform a numerical comparison, on the synthetic sequences of five LR images generated, from $132 \times 132$ fragments of the images showed in Fig. 1, through warping, blurring and downsampling by a factor of $\sqrt{P}=2$. 


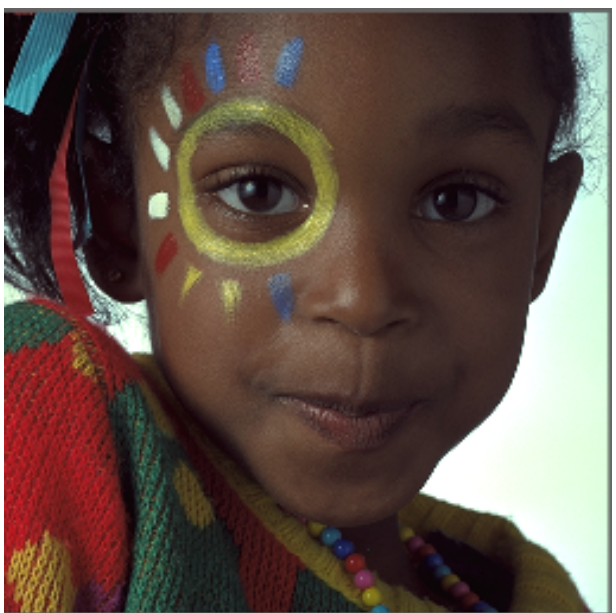

(a)

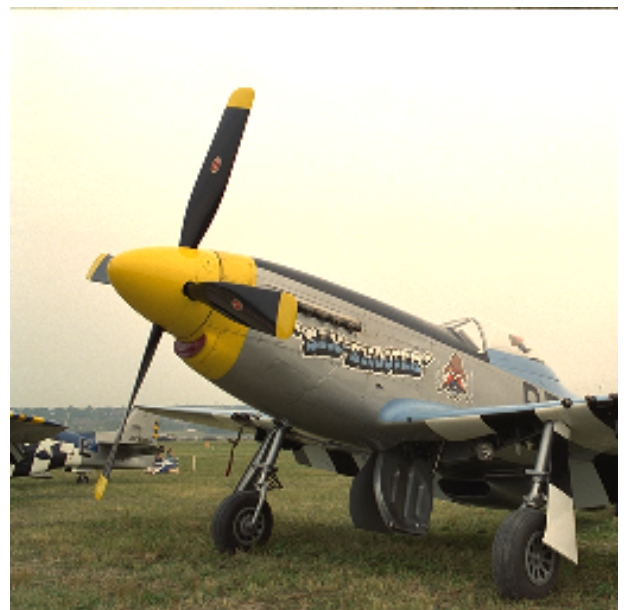

(b)

Fig. 1: Images used in the synthetic experiments.

The warping consisted of translations of $(0,0)^{t},(0,0.5)^{t}$, $(0.5,0)^{t},(1,0)^{t}$ and $(0,1)^{t}$ pixels respectively, and rotations of $0^{\circ}, 3^{\circ},-3^{\circ}, 5^{\circ}$ and $-5^{\circ}$. A $3 \times 3$ uniform PSF has been used for blurring. The LR images obtained after the warping, blurring and downsampling operations are further degraded by additive white Gaussian noise at SNR levels of $10 \mathrm{~dB}, 15 \mathrm{~dB}$, $20 \mathrm{~dB}$ and $25 \mathrm{~dB}$. At each SNR level, ten noise realizations have been utilized.

In this experiment, the quality of the different restored HR images has been measured in terms of the Peak Signalto-Noise Ratio (PSNR), which is defined as PSNR = $10 \log _{10} \frac{N P}{\|\hat{\mathbf{x}}-\mathbf{x}\|^{2}}$, where $\hat{\mathbf{x}}$ and $\mathbf{x}$ are the estimated and original HR images, respectively, with their pixel values normalized to lie in the interval $[0,1]$.

Fig. 2 shows a quantitative comparison in terms of PSNR, of the restorations of the images in Fig. 1 at different noise levels, obtained using the following methods: 1) bicubic interpolation (denoted by $B B C$ ), 2) the SR method in [8] (denoted by ZMT), which is based on backprojection with median filtering, 3) the robust SR method in [9] (denoted by RSR), which is based on bilateral TV priors, 4) the variational SR method using a TV prior in [10] (denoted by TV), 5) the variational SR method in [4] based in a combination of $\ell 1$ and SAR priors (denoted by LISAR), and our proposed algorithm 1 using the filter combinations 6) NF2, 7) NF3, 8) NF4 and 9) NF5. It can be observed in Fig. 2, that the proposed method provides better results than the other methods under comparison, except for the NF2 filter combination at high noise levels.

Let us finally perform a qualitative study of the performance of the proposed method with two real sets of LR observations from a Sony Nex 5 digital camera. Two sets of $19100 \times 100$ RAW images have been obtained using an ISO sensitivity of 6400. Afterward, and assuming a $5 \times 5 \mathcal{N}(0,1)$ integration PSF, superresolved images by a factor $\sqrt{P}=2$ were obtained from each sequence using different SR methods.

Fig. 3 and Fig. 4 show the HR reconstructions obtained from these sets of real observations using $B B C, R S R, Z M T$, $T V, L 1 S A R$ and NF5 methods. In both cases, the proposed method suppresses noise better than the other methods, and provides better reconstructions.

\section{CONCLUSIONS}

In this paper the SR image registration and reconstruction problem has been studied, within the Bayesian framework, using a general sparse image prior combination. A new SR method has been proposed, which allows for the automatic estimation of all the problem unknowns using variational techniques. The proposed method performs better than other state of the art SR methods, specially when the NF3, NF4 and NF5 filter combinations are used for the prior.

\section{REFERENCES}

[1] A. K. Katsaggelos, R. Molina, and J. Mateos, Super Resolution of Images and Video. Morgan and Claypool, 2007.

[2] P. Milanfar, Super-Resolution Imaging, ser. Digital Imaging and Computer Vision. Taylor\&Francis/CRC Press (Ed.), 2010.

[3] S. Villena, M. Vega, R. Molina, and A. K. Katsaggelos, "Bayesian superresolution image reconstruction using an 11 prior," in 6th ISPA Proc., 2009, pp. 152-157.

[4] S. Villena, M. Vega, D. Babacan, R. Molina, and A. Katsaggelos, "Bayesian combination of sparse and non sparse priors in image super resolution," Digital Signal Processing, vol. 23, no. 2, pp. 530-541, 2013.

[5] J. A. Palmer, K. Kreutz-Delgado, and S. Makeig, "Strong sub- and supergaussianity," in LVA/ICA, 2010, pp. 303-310.

[6] S. Babacan, R. Molina, M. Do, and A. Katsaggelos, "Blind deconvolution with general sparse image priors," in European Conference on Computer Vision (ECCV). Florence (Italy), September 2012, pp. 341355.

[7] B. Lucas and T. Kanade, "An iterative image registration technique with an application to stereo vision," in Proceedings of Imaging Understanding Workshop, 1981, pp. 121-130.

[8] A. Zomet, A. Rav-Acha, and S. Peleg, "Robust super-resolution," in IEEE CVPR, 2001, pp. 645-650.

[9] S. Farsiu, M. D. Robinson, M. Elad, and P. Milanfar, "Fast and robust multiframe super resolution," IEEE Trans. Image Process., vol. 13 , no. 10, pp. 1327-1344, Oct. 2004.

[10] S. D. Babacan, R. Molina, and A. Katsaggelos, "Variational bayesian super resolution," IEEE Transactions on Image Processing, vol. 20 , no. 4, pp. $984-999,2011$. 


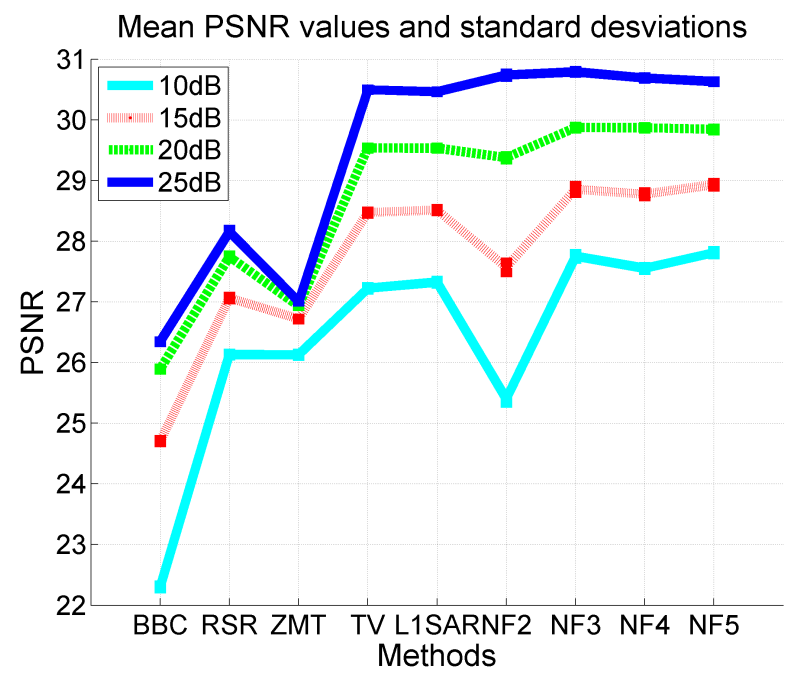

(a)

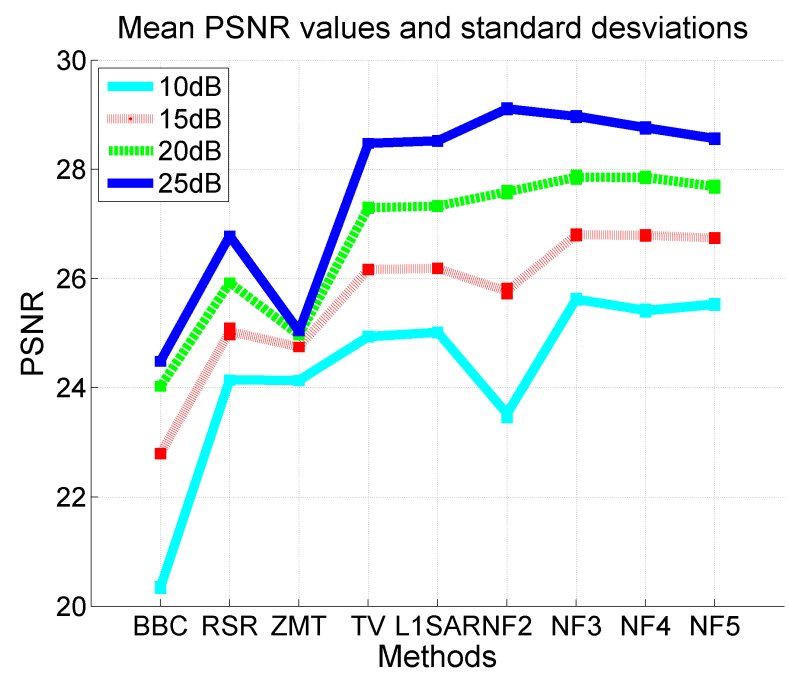

(b)

Fig. 2: Mean PSNR values, and standard deviations, corresponding to different methods and noise levels: (a) for the image in Fig. 1(a), and (b) for the image in Fig. 1(b).

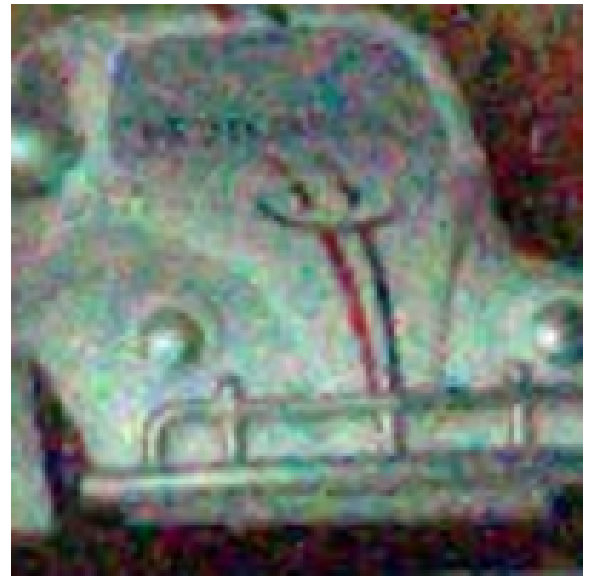

(a)

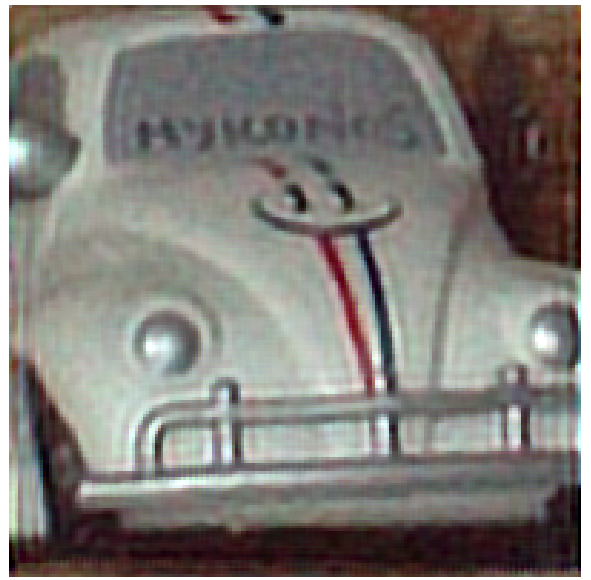

(d)

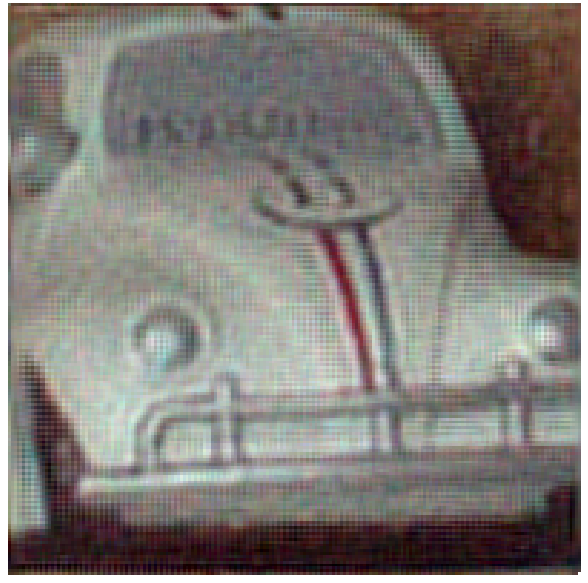

(b)

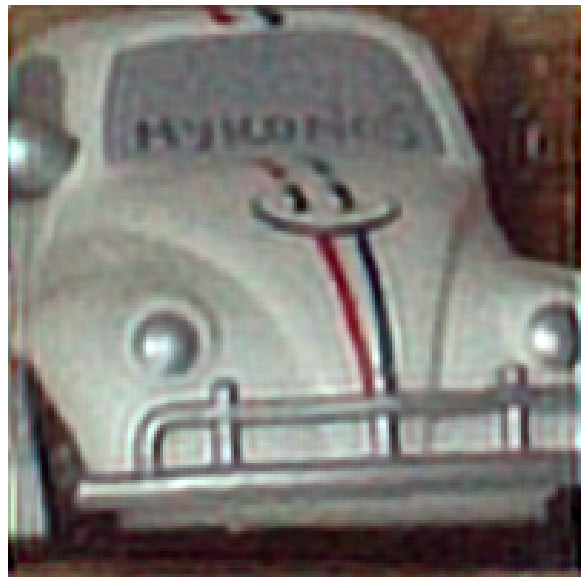

(e)

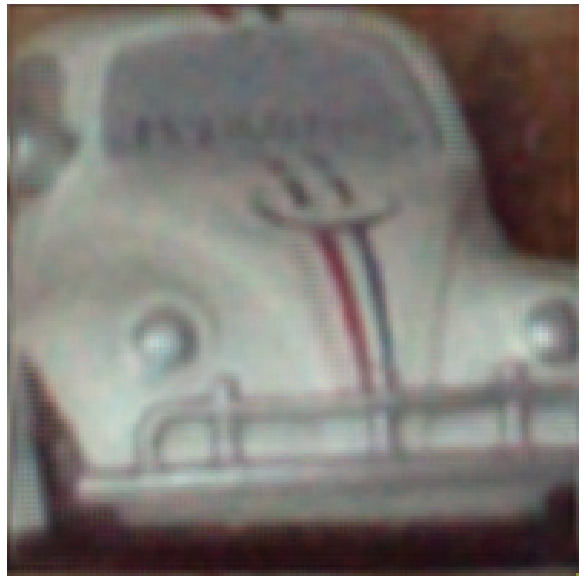

(c)

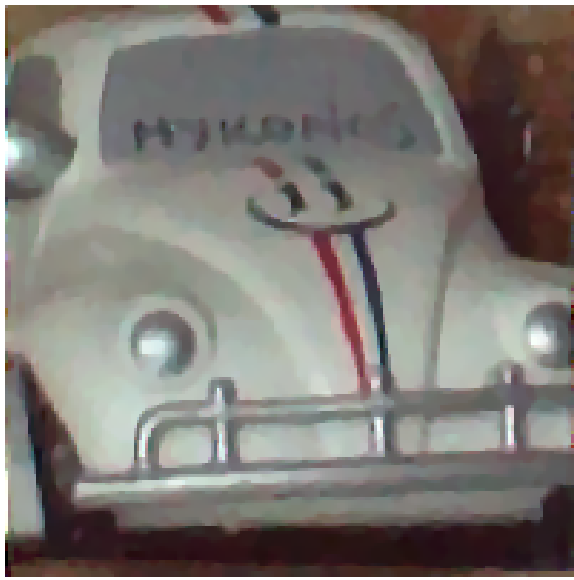

(f)

Fig. 3: HR reconstruction of real images using the following methods: (a) $B B C$, (b) $R S R$, (c) $Z M T$, (d) $T V$, (e) LISAR and (f) NF5 


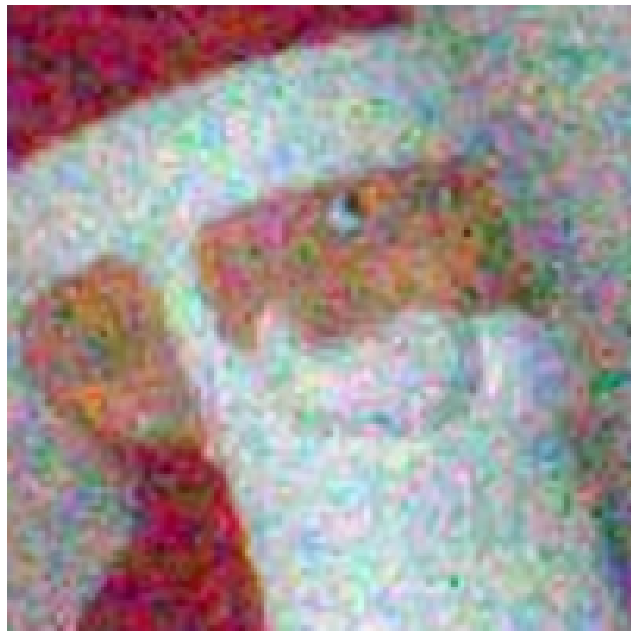

(a)

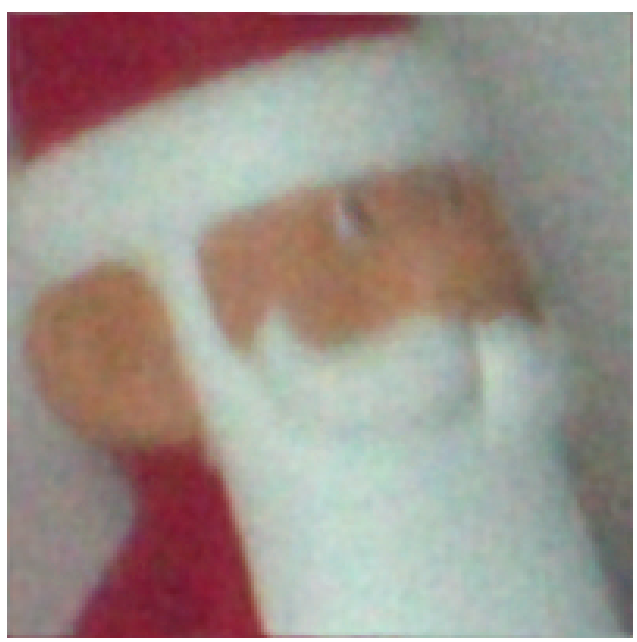

(c)

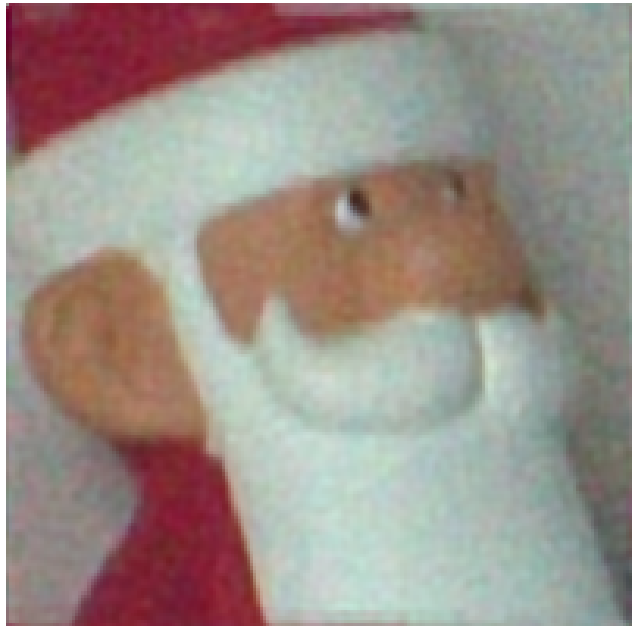

(e)

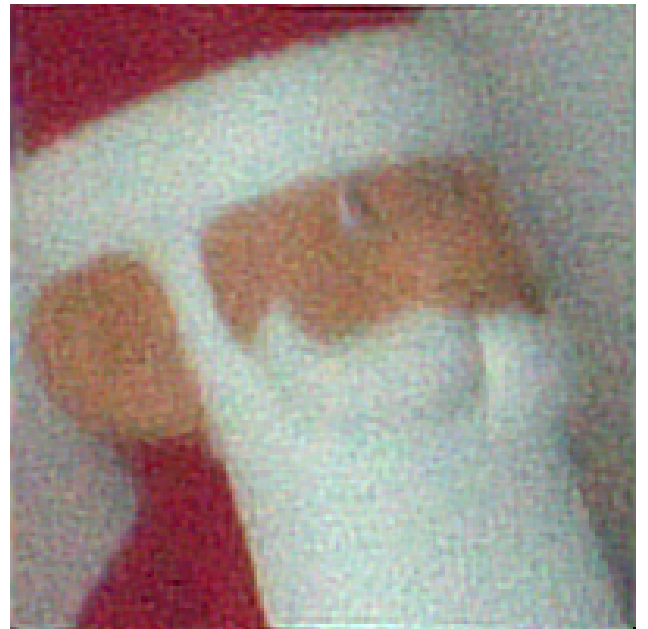

(b)

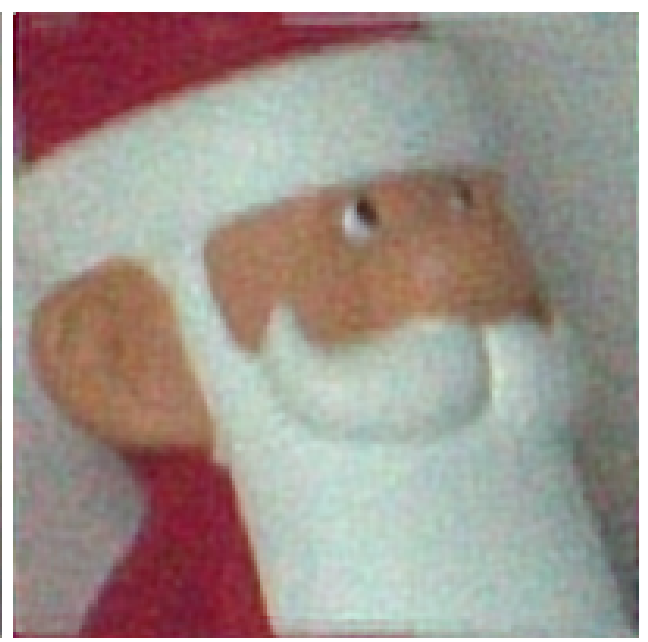

(d)

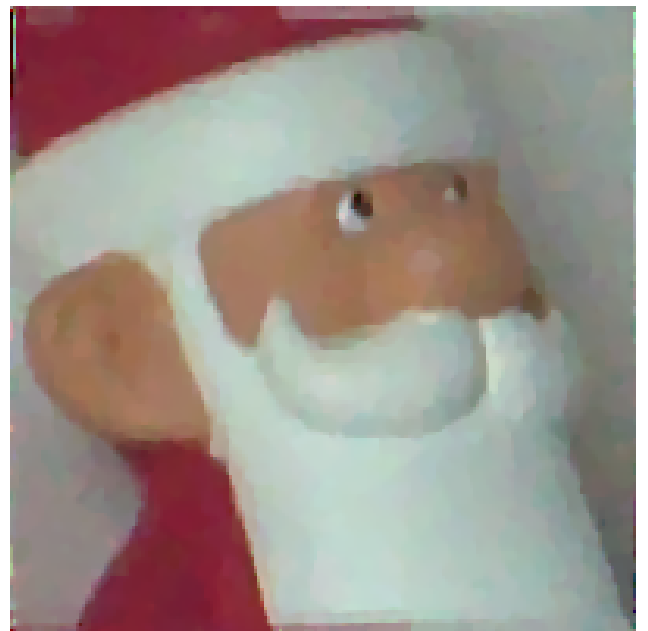

(f)

Fig. 4: HR reconstruction of real images using the following methods: (a) $B B C$, (b) $R S R$, (c) $Z M T$, (d) $T V$, (e) $L 1 S A R$ and (f) NF5 